\title{
Editorial
}

\section{Marine Engineering and Applications}

\author{
Chang-Hua Lien, ${ }^{1}$ Jia-Jang Wu, ${ }^{1}$ Irene Penesis, ${ }^{2}$ Henryk Śniegocki, ${ }^{3}$ and Wen-Jer Chang ${ }^{4}$ \\ ${ }^{1}$ Department of Marine Engineering, National Kaohsiung Marine University, Kaohsiung 811, Taiwan \\ ${ }^{2}$ National Centre for Maritime Engineering and Hydrodynamics, Australian Maritime College, University of Tasmania, \\ Locked Bag 1395, Launceston, TAS 7250, Australia \\ ${ }^{3}$ Department of Navigation, Gdynia Maritime University, Aleja Jana Pawła II 3, 81-345 Gdynia, Poland \\ ${ }^{4}$ Department of Marine Engineering, National Taiwan Ocean University, Keelung 202, Taiwan \\ Correspondence should be addressed to Chang-Hua Lien; chlien@mail.nkmu.edu.tw
}

Received 25 July 2013; Accepted 25 July 2013

Copyright (C) 2013 Chang-Hua Lien et al. This is an open access article distributed under the Creative Commons Attribution License, which permits unrestricted use, distribution, and reproduction in any medium, provided the original work is properly cited.

The number of new ships worldwide continues to grow, and the new technologies are developed to improve the safety and international requirements of ship are increasing as well. This special issue has a strong impact in many fields, like system monitoring and fault diagnostic, corrosion science, vessel's propulsion systems, engineering of vessel's superstructures, green technology and energy, advanced shipboard machinery technology, port engineering, and heat transfer technology, and related topics have been proved to be very useful and powerful tools for solving many kinds of practical problems in marine engineering. This special issue deals with the focused topics in advanced technology.

This special issue contains seventeen papers, the contents of which are summarized as follows.

"Torsional vibrations of a conic shaft with opposite tapers carrying arbitrary concentrated elements" by J. J. Wu provides the exact solution for free torsional vibrations of a conic shaft with opposite tapers carrying any number of rigid disks or/and torsional springs. The technique is significant for evaluating the accuracy of various existing approximate methods. All numerical results obtained from the exact method are compared with those obtained from the conventional finite element method (FEM), and good agreement is achieved.

"Vibrations due to flow-driven repeated impacts" by S. Jeong and N. Baddour reveals that the vibrations caused by repeated impacts of a flow-driven free mass onto a second structure can be used to explain the nature of ice-induced vibrations without requiring a microscopic model of the mechanism of ice failure. Conservation of linear momentum and the coefficient of restitution are used to characterize the nature of the impacts between the two masses.

"Torsion dynamic behavior of the ship hull made out of layered composites" by I. Chirica develops a macroelement model that can be used for torsion dynamic analysis of a ship hull made of composite materials. A simple yet effective ship hull model made out of layered composites was built and tested experimentally to determine the natural frequencies and global vibrations of the model which were then used to verify the results from a newly developed numerical model.

"Risk modelling for passages in approach channel" by L. Smolarek and H. Śniegocki shows how combined methods of multivariate statistics, stochastic processes, and simulation are used to identify and assess the risk measures. This research allows improving ships safety on port approaches, mainly when new berths for large vessels are constructed in approach channels.

"Study on GPS/INS system using novel filtering methods for vessel attitude determination" by X. Chen et al. provides research and simulations whose results show that the determination accuracy and consumption cost of the proposed genetic particle filter (GPF) are better than extended kalman particle filter (EKPF) and traditional KF. This is important for getting more accurate and efficient data received from GPS and INS.

"Application of an integrated model with MCDM and IPA to evaluate the service quality of transshipment port" by C.C. Chou and J.-F. Ding presents multiple-criteria decisionmaking and importance-performance analysis to evaluate 
the service quality of three international ports in Asia. The proposed results show that the integrated model is an appropriate one to evaluate and analyze the service quality of ports.

"Multi objective optimization of coordinated scheduling of cranes and vehicles at container terminals" by S. M. Homayouni and S. H. Tang formulates a mixed integer programming model to optimize the coordinated scheduling of cranes and vehicles in container terminals. A genetic algorithm is developed to solve the problem in reasonable computational time. The most appropriate control parameters for the proposed genetic algorithm are investigated in a medium size numerical test case.

"Processing of instantaneous angular speed signal for detection of a diesel engine failure" by $\mathrm{A}$. Charchalis and $\mathrm{M}$. Dereszewski. presents the method of decomposition of noise of angular speed of a crankshaft using triple decomposition by Savitzky-Golay filter with different width of approximation intervals. Results of smoothing of IAS signals measured during experiments were compared with IAS waveforms obtained by means of mathematical model. In order to evaluate effectiveness of the method, experiments at the test bed, simulating faults of a diesel engine's fuel installation were, carried out.

"Variance and passivity constrained fuzzy control for nonlinear ship steering systems with state multiplicative noises" by W.-J. Chang and B.-J. Huang uses continuous-time TakagiSugeno fuzzy model to represent nonlinear ship steering systems with state multiplicative noises. Some LMI conditions are derived to achieve the variance, passivity, and stability performances.

"Tracking controller design for diving behavior of an unmanned underwater vehicle" by Y.-H. Tseng et al. investigates the almost disturbance decoupling problem of nonlinear uncertain control systems via the fuzzy feedback linearization approach. A feedback control of an unmanned underwater vehicle is proposed in this article. The assumption for small pitch angle has been relaxed for practical applications.

"Automated visual inspection of ship hull surfaces using the wavelet transform" by C. Fernández-Isla et al. develops a new online visual inspection technique from the images obtained from the hull surfaces. A prototype of the automated blasting system has been developed and tested in the Spanish NAVANTIA shipyards.

"Fracturing pressure of shallow sediment in deep water drilling" by C. Yan et al. has developed a theoretical model that considers the excess pore pressure around a wellbore induced by oil and gas drilling. The theoretical model combines the excess pore pressure due to the soil squeezing effect induced by wellbore column pressure with hydraulic fracturing theory, to research the fracturing mechanism of shallow sediment in deep water. A theoretical formula for the fracturing pressure is also developed and compared with measured data.

"An elastoplastic damage constitutive model for cementitious materials under wet-dry cyclic sulfate attack" by D. Chen et al. has investigated the deterioration and sulfateattack process of concrete structures when in a wet-dry cyclic environment. Experiments were performed to determine the mechanical properties of cement subject to wet-dry cyclic sulfate attack under compression and compared to a developed elastoplastic damage constitutive model.

"Fluid flow simulations based on an equation-solving solution gradient strategy" by H.-S. Huang and Y.-H. Hwang introduces a compact and accurate discretization for fluid flow simulations. A supplementary equation and its associated control volume are used to achieve the main goal. Some numerical analyses on simple one-dimensional modeled problems are exposed in this article.

"Chaotic and subharmonic motion analysis of floating ring gas bearing system by hybrid numerical method" by C.-C. Wang et al. analyzes the nonlinear dynamic behavior of a rigid rotor supported by floating ring gas bearing (FRGB) system. The proposed hybrid method can avoid the numerical instability problem suffered by the finite difference scheme at low values of the rotor mass and computational timestep. The results of this article provide an understanding of the nonlinear dynamic behavior of floating ring gas bearing systems characterized by different rotor masses $m_{r}$ and bearing numbers $\Lambda$.

"Remote teleoperated and autonomous mobile security robot development in ship environment" by L.-Y. Chung proposes a wireless remote teleoperated and autonomous mobile security robot based on a multisensor system to monitor the ship/cabin environment. The proposed idea can make one crew member on duty at the cabin is a possible option.

"Ameliorated austenite carbon content control in austempered ductile irons by support vector regression" by C.-Y. Yang et al. uses support vector machine for regression to establish a relationship between the initial carbon concentration of austempered ductile irons after austenization with austenization temperature and alloy contents in austempering processes. A better control of $C_{\gamma}^{0}$ has been proven to be critical in achieving desired microstructures and mechanical properties for ADI, which has been applied in many marine applications.

Of course, the selected topics and papers are not a comprehensive representation of the area of this special issue. Nonetheless, they represent the rich and many-faceted knowledge that we have the pleasure of sharing with the readers.

\section{Acknowledgments}

We would like to express appreciation to the authors for their excellent contributions and patience in assisting us. The hard work of all reviewers on these papers is also very greatly acknowledged.

Chang-Hua Lien Jia-Jang Wu Irene Penesis Henryk Śniegocki Wen-Jer Chang 


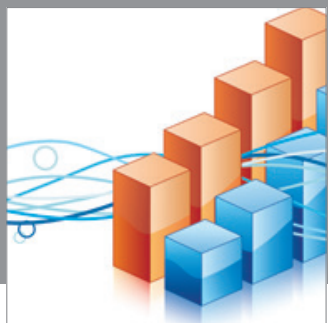

Advances in

Operations Research

mansans

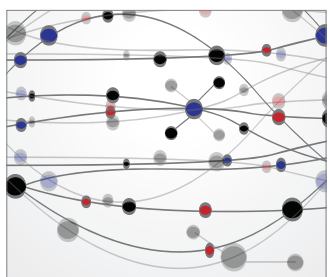

The Scientific World Journal
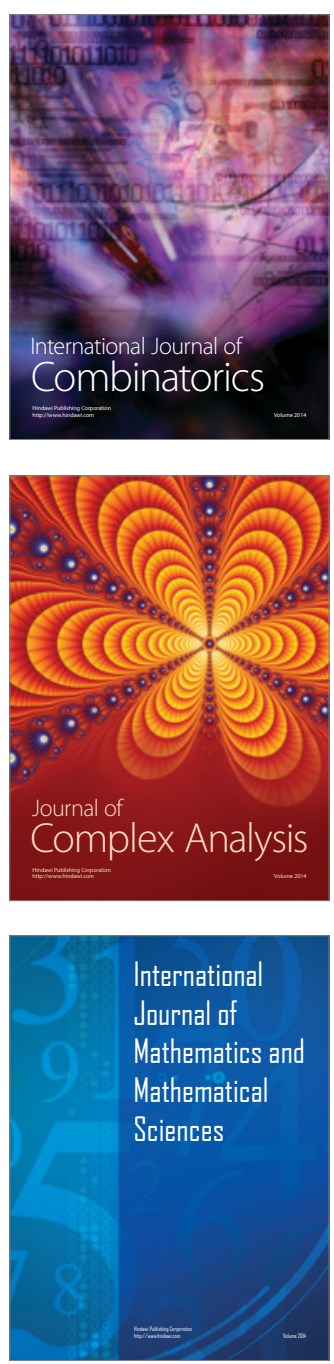
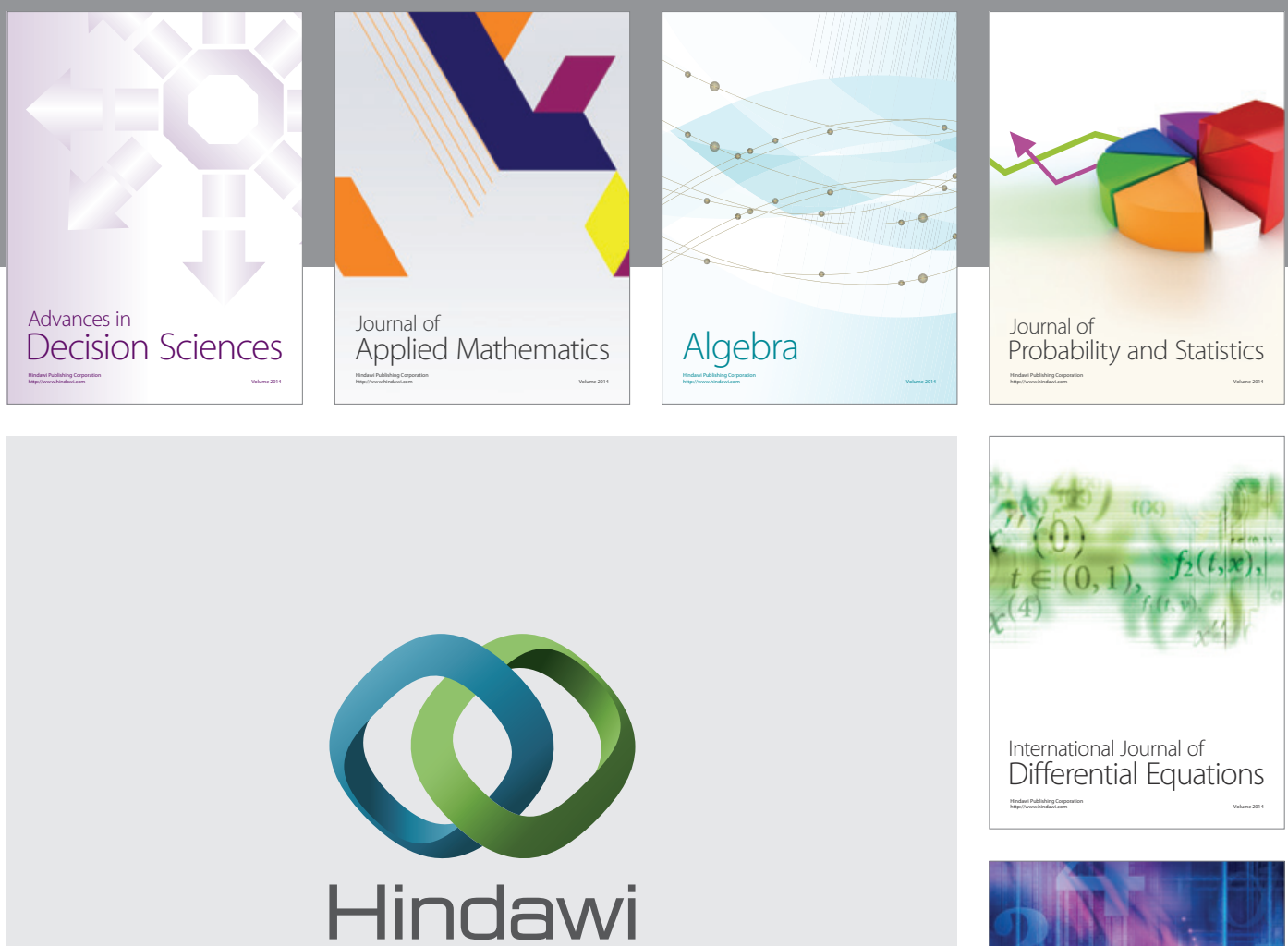

Submit your manuscripts at http://www.hindawi.com
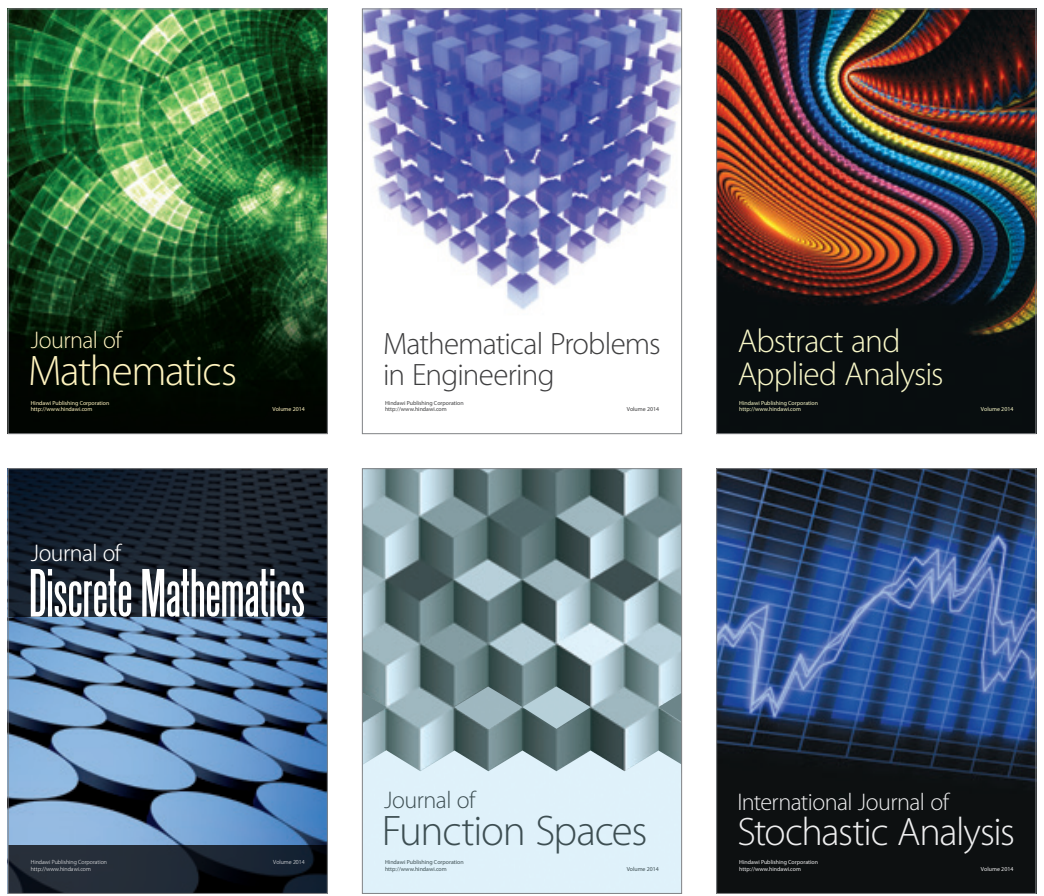

Journal of

Function Spaces

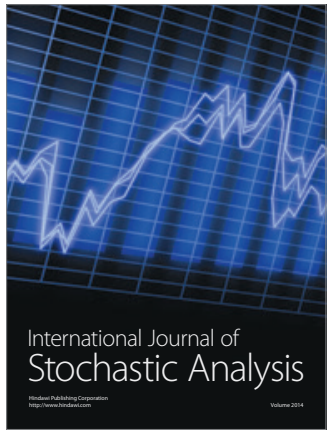

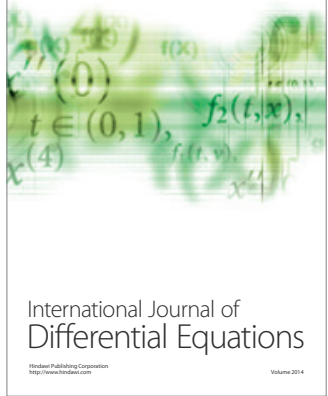
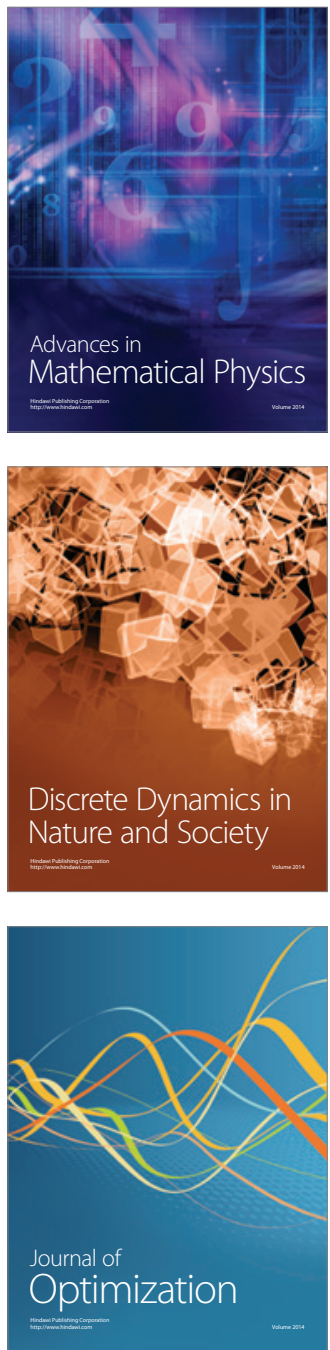